Journal of Engineering and Applied Sciences 14 (Special Issue 1): 3832-3835, 2019

ISSN: 1816-949X

(C) Medwell Journals, 2019

\title{
Extraordinary Learning Machine for Forecast of Wind Force and Moment Coefficients in Marine Vessels
}

\author{
R.N. Raju \\ Department of GMDSS, AMET University, Chennai, India
}

\begin{abstract}
In the current circumstances, seaward exercises are getting progressively vital and marine vessels are common in all the water bodies. This requires a nitty gritty investigation of the impact of ecological powers of the marine structures. This study goes into building up a brought together system to concentrate the impact of wind compel and minutes on seagoing vessels. A neural system approach is created to focus the impact of longitudinal and side strengths of the wind and the yaw moment. The review considers different sorts of oceangoing vessels at various stacking conditions with a sum of 22 marine vessels. Of these, 18 are utilized to prepare a troupe of Extreme Learning Machine (ELM) neural system. The system, therefore, created is tried for speculation on two new sorts of vessels at two unique stacking conditions. In this manner, the generated model is fit for anticipating the wind compel and minute coefficients, independent of the sort of vessel utilized. An ensemble of extreme learning machine each with input parameters initialized at different regions of the entry space are trained with all training samples. For each sample, the ELM that produces the least mean square error is identified and the output of that ELM is considered as the output for that sample. Thus, the randomness of the initialization in ELM is exploited to achieve superior generalization performance. Performance study to predict the wind force and moment coefficients of seagoing vessels show that the ensemble of ELM has excellent prediction performance, compared to state of the art results for this problem.
\end{abstract}

Key words: Wind force and moment drag coefficient, Extreme Learning Machine (ELM), Single hidden Layer Feedforward neural Network (SLFN), Support Vector Regression (SVR), Multilayer Perceptron (MLP), dynamic compnents

\section{INTRODUCTION}

There has been a significant increment in the seaward exercises in the current circumstances. This requires productive dynamic situating methods to bridge the oceangoing vessels securely. Marine vessels are unbending body structures, whose development is impacted by static and dynamic components. Static elements are bodies very still or those moving at consistent speeds while active components have capricious quickened movement. Such powerful elements incorporate hydrodynamic powers, minutes and unsettling genetic influences, for example, the wind, wave and streams (Isherwood, 1973; Haddara and Soares, 1999).

Multivariable functional interpolation and adaptive networks are presented by Broomhead and Lowe (1988), Igelnik and Pao (1995). Complex systems, stochastic choice of basis functions in adaptive function approximation and the functional-link net. The study of Huang (2003) describes learning capability and storage capacity of two-hidden-layer feedforward networks. A survey on extreme learning machines has been enlightening by Huang et al. (2011).

The convention for the wind forces and moments acting on a marine vessel is presented in Fig. 1 (Blendermann, 1994). The longitudinal force, the side strength and yaw moments are represented by $F_{x}, F_{y}$ and $\mathrm{N}$, respectively. B. represents the angle between the wind

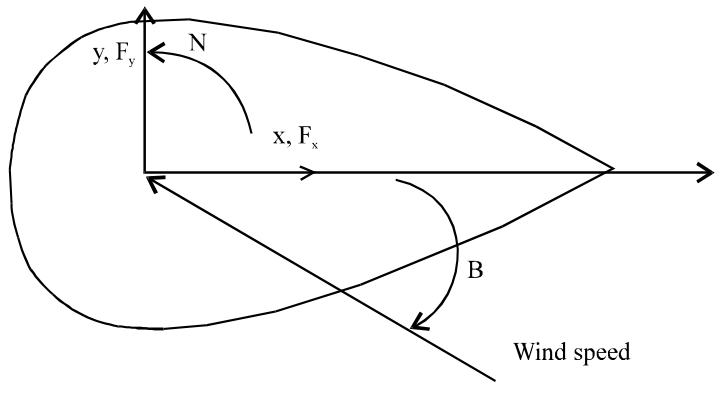

Fig. 1: Forces and moment on marine vessels 
speed and ship bow The high-efficiency renewable PV inverter topology is described. Firefly algorithm is portrayed for reactive power optimization in marine.

This study also described in decomposition for 3D electromagnetic modeling. Earth, planets and space (Xiong, 1999). Current channeling in time-domain airborne electromagnetic data (Reid and Macnae, 2000). Wind forces $\left(F_{\mathrm{z}}\right.$ and $\mathrm{F}_{\mathrm{y}}$ in Newton) and moment ( $\mathrm{MN}$ in Newton-metre) acting on a marine vessel is determined by:

$$
\begin{aligned}
\mathrm{F}_{\mathrm{x}} & =\frac{1}{2} \mathrm{C}_{\mathrm{x}}(\gamma \mathrm{r}) \rho \mathrm{a}_{s_{\mathrm{x}}^{2}} \mathrm{~A}_{\mathrm{TR}} \\
\mathrm{F}_{\mathrm{y}} & =\frac{1}{2} \mathrm{C}_{\mathrm{y}}(\gamma \mathrm{r}) \rho \mathrm{a}_{s_{\mathrm{z}}^{2}} \mathrm{~A}_{\mathrm{LA}} \\
\mathrm{MN} & =\frac{1}{2} \mathrm{C}_{\mathrm{N}}(\gamma \mathrm{r}) \rho \mathrm{a}_{s_{\mathrm{s}}^{2}} \mathrm{~A}_{\mathrm{LA \lambda}}
\end{aligned}
$$

Where:

$$
\begin{array}{ll}
\mathrm{C}_{\mathrm{x}} \text { and } \mathrm{C}_{\mathrm{y}} & =\text { The empirical force coefficients } \\
\mathrm{C}_{\mathrm{N}} & =\text { The observed moment coefficient } \\
& =\text { The density of air } \\
\mathrm{e}_{\mathrm{a}}\left(\mathrm{kg} / \mathrm{m}^{3}\right) & =\text { The wind velocity in knots } \\
\mathrm{S}_{\mathrm{r}} & =\text { The transverse and lateral projected } \\
\text { ATR }\left(\mathrm{m}^{2}\right) & \text { area } \\
\text { and ALA }\left(\mathrm{m}^{2}\right) & \\
& =\text { The overall length of the ship }
\end{array}
$$

\section{MATERIALS AND METHODS}

Ensemble of extreme learning machine predictor: ELM is a feedforward neural network, whose input parameters are chosen randomly and the output weights are estimated analytically. For any data set with $\mathrm{L}$ training samples, $\left\{\left(\mathrm{u}_{1}, \mathrm{v}_{1}\right),\left(\mathrm{u}_{\mathrm{t}}, \mathrm{v}_{\mathrm{t}}\right),\left(\mathrm{u}_{\mathrm{L}}, \mathrm{v}_{\mathrm{L}}\right)\right\}$; $u_{t^{-}-} m, v_{t_{-}-} n$, the regression problem is defined as approximating the functional relationship $\mathrm{f}: \mathrm{u}_{\mathrm{r}} \mathrm{vet}$ as accurately as possible, enabling output prediction for new samples with better accuracy. An ELM with $m$ input neurons and $\mathrm{n}$ output neurons is required to solve this regression problem.

The summed up execution of ELM relies on upon the introduction of the focuses $(\mu)$ and width $\left({ }_{0}\right)$. Subsequently, via. preparing various ELM in different locales of the info space, one can successfully enhance the overall forecast execution. The square outline of the proposed outfit of ELM is introduced in Fig. 2. We initially prepare M ELM to be specific, $\{E L M[1], E L M[2], E L M[M]\}$ with every one of the examples the preparation informational index. The Gaussian focus and width parameters of the neurons

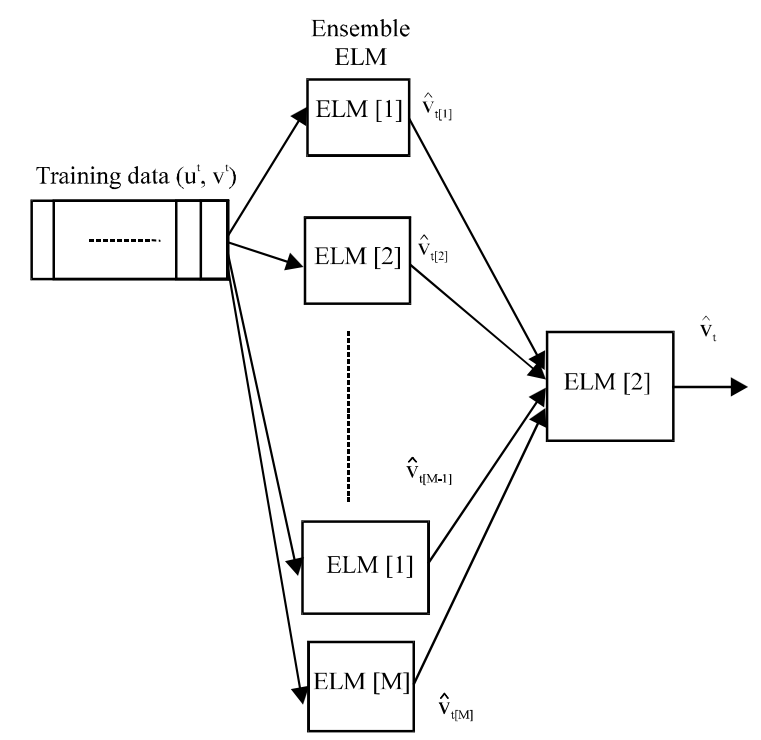

Fig. 2: Block diagram of the ensemble of ELM

in the shrouded layer for every ELM is introduced in various districts of the info space. The ELM that exhibits the base mean square mistake for each example is recognized. The systems that add a big mistake for every one of the specimens are disposed of from the outfit. The yield of every ELM in the group is then joined utilizing the min (MSE) work which processes the base mean square blunder for each example.

\section{RESULTS AND DISCUSSION}

The execution consequences of the wind force and moment coefficient expectation for the Tanker in stabilizer condition at different approaches (r) is displayed in Fig. 3. Figure 3a, b show the execution comes about for the forecast of longitudinal wind drive coefficient; side wind compels coefficient and the yaw minute coefficient, individually. From the outcomes, it can be watched that the execution of the outfit of ELM is superior to that of the MLP, SVR and ELM and predicts the coefficients precisely as acquired from actual methodologies.

Figure $4 \mathrm{a}, \mathrm{b}$ presents the coefficient prediction results for the Gas tanker in ballast condition at various angles of attack (r). The longitudinal factor prediction results are shown in the side force coefficient prediction results in Fig. $4 \mathrm{~b}$ and the yaw moment coefficient prediction results are presented. The performance results indicates that the ensemble of ELM predicts the factors accurately. Compared to 

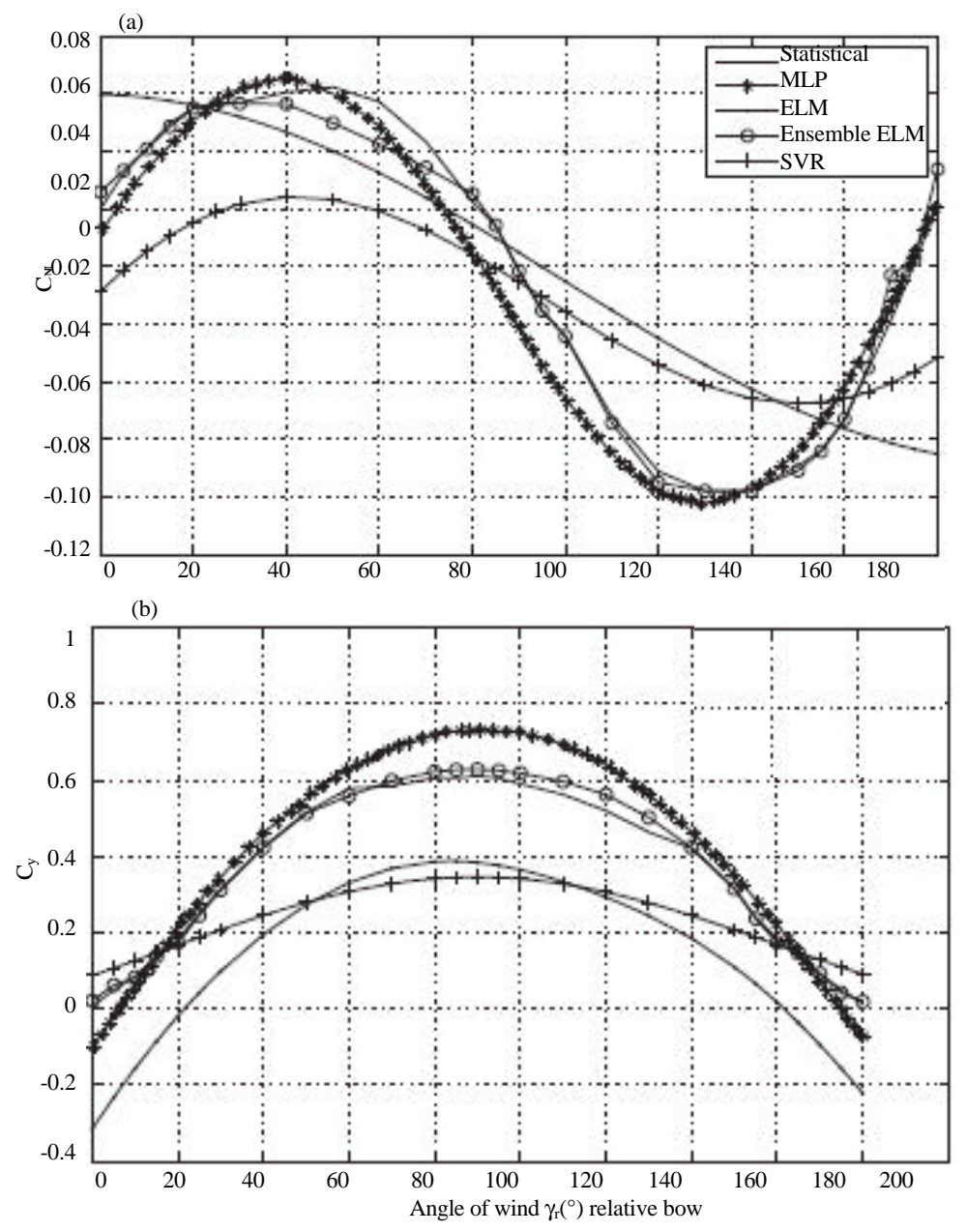

Fig 3: a) Yaw moment coefficient of tanker (ballast) and b) Side Force coefficient of tanker (ballast)

(a)

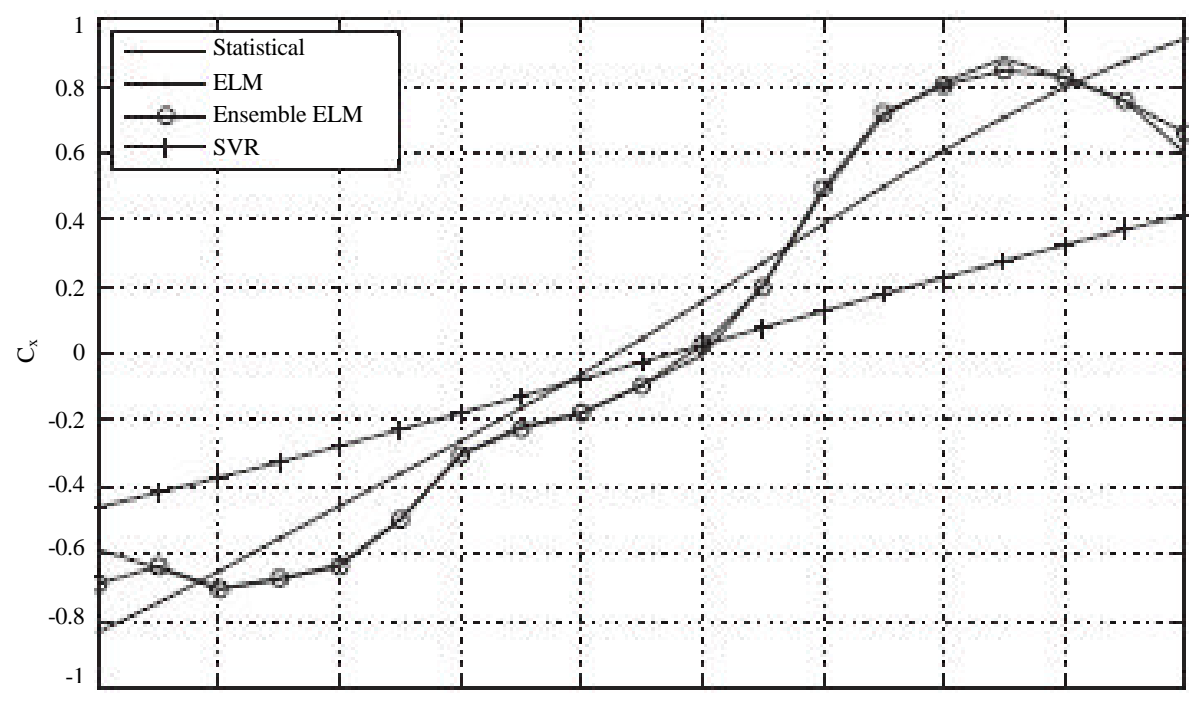

Fig. 4: Continue 


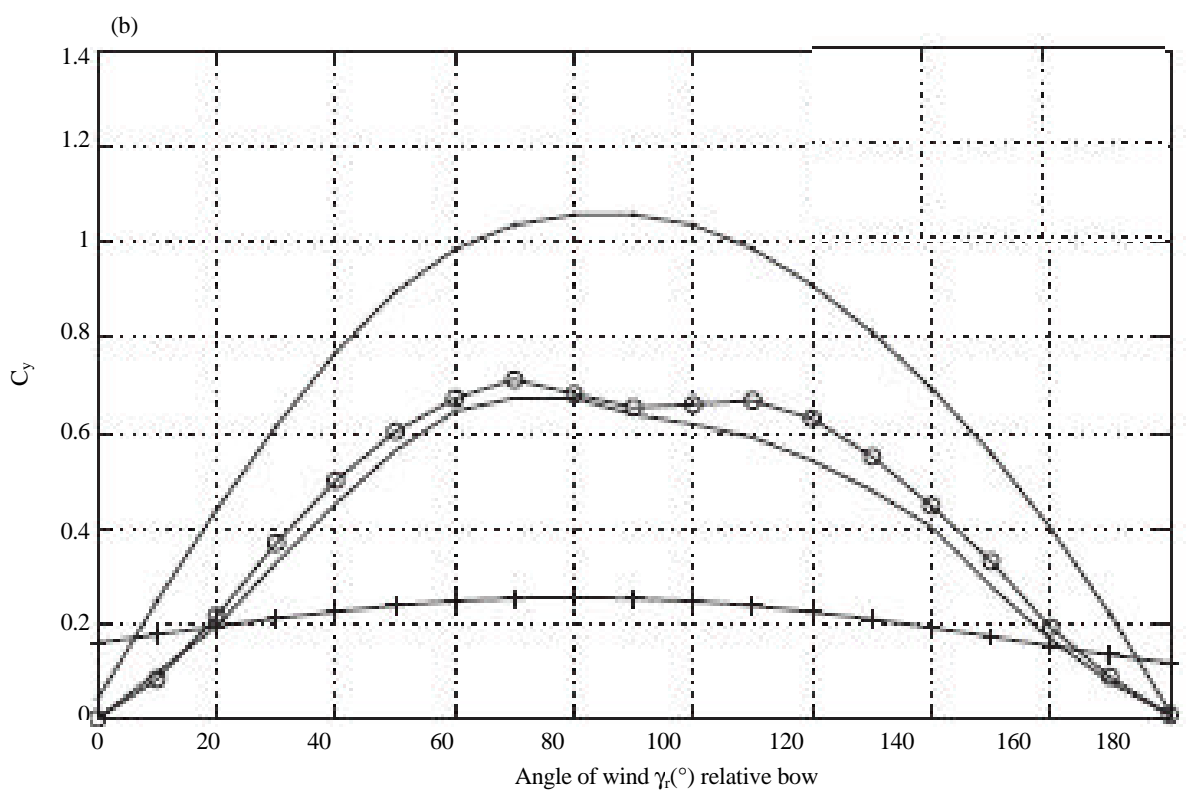

Fig. 4: a) Longitudinal force coefficient of gas tanker (ballast) and b) Side force coefficient of gas tanker (ballast)

MLP, SVR and a single ELM, ensemble ELM is more accurate and the curve follows that of the statistical approach.

\section{CONCLUSION}

In this study, we display a brought together system to foresee the wind powers and the minute factor of various sorts of oceangoing vessels at different stacking conditions. A group of the extraordinary learning machine is introduced to gauge the longitudinal and side drive coefficients and the yaw minute factor. The gathering of ELM is prepared to evaluate the factors of 10 distinctive sort of oceangoing vessels at various stacking conditions, i.e., holder dispatch, payload, driving a base ship, penetrate send, voyage transport, angle cutter, cargo ship, research vessel, speed watercraft and offshore supply vessel. The execution of the group of ELM was tried with a tanker and gas truck at two distinctive stacking conditions. Execution contemplate brings about the correlation with the cutting edge strategy for coefficient expectation demonstrate the prevalent speculation capacity of the outfit of ELM.

\section{REFERENCES}

Blendermann, W., 1994. Parameter identification of wind loads on ships. J. Wind Eng. Ind. Aerodyn., 51: 339-351.

Broomhead, D.S. and D. Lowe, 1988. Multivariable functional interpolation and adaptive networks. Complex Syst., 2: 321-355.

Haddara, M.R. and C.G. Soares, 1999. Wind loads on marine structures. Mar. Struct., 12: 199-209.

Huang, G.B., 2003. Learning capability and storage capacity of two-hidden-layer feedforward networks. IEEE. Trans. Neural Netw., 14: 274-281.

Huang, G.B., D.H. Wang and Y. Lan, 2011. Extreme learning machines: A survey. Int. J. Mach. Learn. Cybern., 2: 107-122.

Igelnik, B. and Y.H. Pao, 1995. Stochastic choice of basis functions in adaptive function approximation and the functional-link net. IEEE. Trans. Neural Netw., 6: 1320-1329.

Isherwood, R.M., 1973. Wind resistance of merchant ships. RNNA. Supplementary Pap., 115: 327-338.

Reid, J. and J. Macnae, 2000. Current channelling in time-domain airborne electromagnetic data. Explor. Geophys., 31: 150-157.

Xiong, Z., 1999. Domain decomposition for 3D electromagnetic modeling. Earth Planets Space, 51: 1013-1018. 